\title{
Contributions of Coding Efficiency of Temporal-Structure and Level Information to Lateralization Performance in Young and Early- Elderly Listeners
}

\author{
Atsushi Ochi, Tatsuya Yamasoba and Shigeto Furukawa
}

\begin{abstract}
The performance of a lateralization task based on interaural time or level differences (ITDs or ILDs) often varies among listeners. This study examined the extent to which this inter-listener variation could be accounted for by the coding efficiency of the temporal-structure or level information below the stage of interaural interaction. Young listeners (20s to 30s) and early-elderly (60s) listeners with or without mild hearing loss were tested. The ITD, ILD, TIME, and LEVEL tasks were intended to measure sensitivities to ITDs, ILDs, the temporal structure of the stimulus encoded by the neural phase locking, and the stimulus level, respectively. The performances of the ITD and ILD tasks were not significantly different between the age groups, while the elderly listeners exhibited significantly poorer performance in the TIME task (and in the LEVEL with a high-frequency stimulus only) than the young listeners. Significant correlations were found between thresholds for the ILD and LEVEL tasks with low- and high-frequency stimuli and for the ITD and TIME tasks for the high-frequency stimulus, implying peripheral coding efficiency as a major factor determining lateralization performance. However, we failed to find a correlation between the ITD and TIME tasks for the low-frequency stimulus, despite a large range of threshold values in the TIME task. This implies that in a low frequency region, the peripheral coding efficiency of the stimulus temporal structure is a relatively minor factor in the ITD-based lateralization performance.
\end{abstract}

Keywords Interaural time difference - Interaural level difference - Temporal structure $\cdot$ Intensity coding $\cdot$ Inter-individual variation $\cdot$ Aging

\footnotetext{
S. Furukawa $(\square)$

Human Information Science Laboratory, NTT Communication Science Laboratories, NTT

Corporation, Atsugi, Japan

e-mail: furukawa.shigeto@lab.ntt.co.jp
}

A. Ochi $\cdot$ T. Yamasoba

Department of Otolaryngology, Faculty of Medicine, University of Tokyo, Tokyo, Japan

e-mail: ochia-tky@umin.ac.jp

T. Yamasoba

e-mail: tyamasoba-tky@umin.ac.jp

(C) The Author(s) 2016

P. van Dijk et al. (eds.), Physiology, Psychoacoustics and Cognition in Normal and Impaired Hearing, Advances in Experimental Medicine and Biology 894,

DOI 10.1007/978-3-319-25474-6 3 


\section{Introduction}

Interaural time and level differences (ITDs and ILDs) are the major cues for horizontal sound localization. Sensitivities to ITDs and ILDs, evaluated by lateralization tasks, often vary markedly among listeners. Lateralization performance based on ITDs and ILDs should reflect not only the listener's ability to compare time and level information, respectively, between ears but also the efficiency of encoding information about the temporal structure and intensity of stimuli at stages below binaural interactions in auditory processing. Our earlier study attempted to evaluate the relative contributions of these processing stages to the inter-listener variability in lateralization performance, by comparing individual listeners' monaural sensitivities to the temporal structure and intensity of a sound stimulus with their ITD and ILD sensitivities (Ochi et al. 2014). The results showed significant correlation of ILD discrimination thresholds with thresholds for monaural level-increment detection task. This could be interpreted as indicating that the inter-individual differences in ILD sensitivity could be (partially) accounted for by the level coding efficiency at stages before binaural interaction. Similarly, ITD discrimination thresholds were found to correlate with the listeners' sensitivities to the temporal structure of monaural stimuli, when the stimuli were in high frequency range (around $4000 \mathrm{~Hz}$ ). However, we failed to find a positive correlation for stimuli in low-frequency range (around $1100 \mathrm{~Hz}$ ).

The present study extends our earlier study (Ochi et al. 2014) by incorporating early-elderly listeners under essentially the same experimental settings. We adopted early-elderly listeners because generally they would exhibit deteriorated sensitivities to temporal structures of stimuli, while their audiometric thresholds remain within a normal to mildely-impaired range. We first examined the effects of age on the performance of individual tasks. We then analysed correlations, as in the earlier study, between task performances. It has been reported that sensitivities to the temporal structure and intensity of stimuli decline with age (e.g., Hopkins and Moore 2011). A population including young and elderly listeners would therefore exhibit a large variability of thresholds in the monaural tasks, which would lead to improved sensitivity of the correlation analyses and provide further insights as to the roles of monaural processing in ITD or ILD discrimination. Supplemental data were also obtained to evaluate underlying mechanisms for the monaural tasks.

As in the earlier study, we measured listeners' performances in four basic tasks, namely ITD, ILD, TIME, and LEVEL tasks, which would reflect sensitivities to ITDs, ILDs, the temporal structure, and the level change of stimuli, respectively. Low- and high-frequency stimuli were tested, which were centred at around 1100 and $4000 \mathrm{~Hz}$, respectively. Supplementary experiments measured frequency resolution (FRES task), frequency discrimination limens (FDISC task), and (for lowfrequency stimulus only) the discrimination threshold of Huggins pitch (HUGGINS task; Cramer and Huggins 1958). 


\section{Methods}

\subsection{Listeners}

Forty-three adults participated in the experiment. All gave written informed consent, which was approved by the Ethics Committee of NTT Communication Science Laboratories. Those included 22 normal-hearing young listeners (referred to as the YNH group; 10 males and 12 females; 19-43 years old, mean 32.0) and 21 elderly listeners (11 males and 10 females; 60-70 years old, mean 63.0). The data from the YNH listeners have been represented in the earlier study (Ochi et al. 2014). In the analyses, the elderly listeners were further divided into normal-hearing (referred to as ENH) and hearing-impaired (EHI) groups. Listeners with audiometric thresholds of $<30 \mathrm{~dB} \mathrm{HL}$ at all the frequencies between 125 and $4000 \mathrm{~Hz}$ in both ears were classified as normal-hearing; otherwise, as (mildly) hearing-impaired. For the FDISC and HIGGINS tasks, a subset of $\mathrm{YNH}$ listeners $(\mathrm{N}=12)$ and all the elderly listeners participated. Table 1 summarizes the means and standard deviations of hearing levels obtained by pure-tone audiometry.

\subsection{Stimuli}

Stimuli were presented to the listener through headphones. Except for the binaural tasks (i.e., ITD, ILD, and HUGGINS tasks), the stimuli were presented to the right ear.

The main four tasks (namely, ITD, ILD, TIME, and LEVEL tasks) employed two types of stimuli, referred to as the low- and high-frequency stimuli, which were identical to those used in our earlier study (Ochi et al. 2014) and are thus only described briefly here. The stimuli were designed to assess the listener's ability to use information on the basis of neural phase-locking to the stimulus temporal structure, respectively, in the ITD and TIME tasks. Essentially the same stimuli were also used in the ILD and LEVEL tasks. The low-frequency stimulus was a spectrally shaped multicomponent complex (SSMC), which was a harmonic complex with a fundamental frequency $\left(F_{0}\right)$ of $100 \mathrm{~Hz}$. The spectral envelope had a flat passband and sloping edges $\left(5 \times F_{0}\right.$ centered at $\left.1100 \mathrm{~Hz}\right)$. The overall level of the complex was $54 \mathrm{~dB}$ SPL. Threshold equalizing noise, extending from 125 to $15,000 \mathrm{~Hz}$,

Table 1 Mean and standard deviations of hearing levels for the three listener groups. Columns represent, from left to right, averages across all the frequencies, $1000-\mathrm{Hz}$ tone, and $4000-\mathrm{Hz}$ tone, respectively

\begin{tabular}{l|l|l|l}
\hline & \multicolumn{3}{|l}{ Hearing level $(\mathrm{dB})$ mean \pm standard deviation } \\
\hline & $125-4000 \mathrm{~Hz}$ & $1000 \mathrm{~Hz}$ & $4000 \mathrm{~Hz}$ \\
\hline YNH $(\mathrm{N}=22)$ & $8.1 \pm 6.5$ & $5.5 \pm 5.6$ & $3.1 \pm 6.5$ \\
\hline ENH $(\mathrm{N}=12)$ & $13.0 \pm 6.1$ & $10.2 \pm 7.0$ & $11.7 \pm 6.2$ \\
\hline EHI $(\mathrm{N}=9)$ & $21.1 \pm 13.4$ & $11.9 \pm 7.1$ & $35.3 \pm 18.8$ \\
\hline
\end{tabular}


was added. The high-frequency stimulus was a "transposed stimulus," which was a $4-\mathrm{kHz}$ tone carrier amplitude-modulated with a half-wave rectified $125-\mathrm{Hz}$ sinusoid (Bernstein and Trahiotis 2002). It is considered that the auditory-nerve firing is phase locked to the modulator waveform, which provides the cue for judging the ITD and modulation rate of the stimulus. The overall level of the transposed stimulus was set to $65 \mathrm{~dB}$ SPL. A continuous, low-pass filtered Gaussian noise was added to prevent the listener from using any information at low spectral frequencies (e.g., combination tones).

Stimuli used for supplementary tasks (namely, FRES and FDISC tasks) involved tone-burst signals at frequencies of 1100 and $4000 \mathrm{~Hz}$. Specifically to these two tasks, the low- and high-frequency stimuli refer to the 1100 - and $4000-\mathrm{Hz}$ tones, respectively. The frequency band of interest in the HUGGINS task (another supplementary task) was centred at $1100 \mathrm{~Hz}$. Other details about the stimuli for the FRES, FDISC, and HUGGINS tasks are described in the next subsection.

\subsection{Procedures}

\subsubsection{General Procedure}

A two-interval two-alternative forced-choice (2I-2AFC) method was used to measure the listener's sensitivities to stimulus parameters. Feedback was given to indicate the correct answer after each response. The two-down/one-up adaptive tracking method was used to estimate discrimination thresholds.

\subsubsection{Task Specific Procedures}

\subsubsection{ITD Task}

In a $2 \mathrm{I}-2 \mathrm{AFC}$ trial, stimuli in the two intervals had ITDs of $+\triangle I T D / 2$ and $-\triangle I T D / 2$ $\mu \mathrm{s}$. Each stimulus was $400-\mathrm{ms}$ long, including 100 -ms raised-cosine onset and offset ramps, which were synchronized between the two ears. The listeners were required to indicate the direction of the ITD change between the two intervals on the basis of the laterality of sound images.

\subsubsection{ILD Task}

Similarly to the ITD task, stimuli in the two intervals had ILDs of $+\triangle I L D / 2$ and $-\triangle I L D / 2 \mathrm{~dB}$. Each stimulus was 400 -ms long, including 20 -ms raised-cosine onset and offset ramps. The listeners were required to indicate the direction of the ILD change between the two intervals on the basis of the laterality of sound images. 


\subsubsection{TIME Task}

For the low-frequency stimulus, the listeners were required to detect a common upward frequency shift $(\Delta f \mathrm{~Hz})$ imposed on the individual components of the SSMC with the spectral envelope remaining unchanged (Moore and Sek 2009). It was assumed that the listeners based their judgments on pitch changes, reflecting the temporal fine structure encoded as the pattern of neural phase locking. The "signal" and "non-signal" intervals in the $2 \mathrm{I}-2 \mathrm{AFC}$ method contained RSRS and RRRR sequences, respectively, where $\mathrm{R}$ indicates the original SSMC and S indicates a frequency-shifted SSMC. For the high-frequency stimulus, the listener's task was to discriminate the modulation frequencies of the transposed stimuli between $f_{\mathrm{m}}$ $(=125 \mathrm{~Hz})$ and $f_{\mathrm{m}}+\Delta f \mathrm{~Hz}$, referred to as $\mathrm{R}$ and $\mathrm{S}$, respectively. Each $\mathrm{R}$ and $\mathrm{S}$ had a duration of $100 \mathrm{~ms}$, including 20 -ms raised-cosine ramps. The threshold was expressed as $\Delta f / f_{0}$ or $\Delta f / f_{\mathrm{m}}$ for the low- or high-frequency stimuli, respectively. When adaptive tracking failed to converge within this limit, trials with a shift of $0.5 F_{0}$ were repeated 30 times. In that case, the proportion of correct trials was converted to $d$ ', and then the "threshold" was derived on the assumption that $d$ ' is proportional to the frequency shift (Moore and Sek 2009).

\subsubsection{LEVEL Task}

In a $2 \mathrm{I}-2 \mathrm{AFC}$ trial, the listeners were required to indicate an interval containing a 400-ms-long SSMC or a transposed stimulus whose central 200-ms portion (including 20 -ms raised-cosine ramps) was incremented in level by $\Delta L \mathrm{~dB}$, while the other non-signal interval contained an original SSMC or a transposed stimulus.

\subsubsection{FRES Task}

The notched-noise masking method (Patterson et al. 1982) was employed to evaluate frequency selectivity. The signals were pure-tone busts centred at 1100 or $4000 \mathrm{~Hz}$, and the maskers were notched noises with varying notch width $(0,0.05$, $0.1,0.2,0.3$, and 0.4 relative to the signal frequency). The spectrum level within the passband was $40 \mathrm{~dB}$ SPL. A rounded-exponential filter (Patterson et al. 1982) was fitted to the experimental data using a least-square fit. The equivalent rectangular bandwidth $(E R B)$ was then derived from the parameters of the fitted filter.

\subsubsection{FDISC Task}

Frequency difference limens were measured with pure-tone bursts centred at 1100 and $4000 \mathrm{~Hz}$. Similarly to the TIME task, the sequence of RRRR and RSRS was presented, $\mathrm{R}$ and $\mathrm{S}$ representing tone bursts with frequencies of $\mathrm{f}_{\mathrm{c}}$ and $f_{\mathrm{c}}+\Delta f \mathrm{~Hz}$, respectively $\left(f_{\mathrm{c}}=1100\right.$ or $\left.4000 \mathrm{~Hz}\right)$. Each tone burst had $200 \mathrm{~ms}$ of duration with $20 \mathrm{~ms}$ onset-offset ramps. 


\subsubsection{HUGGINS Task}

Bandpass-filtered noises (passband: 250-4000 Hz) with a duration of $200 \mathrm{~ms}$ were used as stimuli. The noise was diotic except for a narrow frequency band centred around $1100 \mathrm{~Hz}\left(f_{\mathrm{c}}\right)$ with an $18 \%$ width around the centre frequency, on which an interaural phase transition was imposed. This stimulus elicits a sensation of pitch corresponding to $\mathrm{f}_{\mathrm{c}}$ (Cramer and Huggins 1958). Similarly to the TIME task, the sequences of RRRR and RSRS were presented, with $\mathrm{R}$ and $\mathrm{S}$ representing tone bursts with frequencies of $\mathrm{fc}$ and $f_{\mathrm{c}}+\Delta f \mathrm{~Hz}$, respectively. The discrimination threshold was expressed as $\Delta f / f_{\mathrm{c}}$.

\subsection{Data Analyses}

MATLAB with Statistical Toolbox was used for statistical analyses of the data. For the ITD, TIME, LEVEL, FDISC, and HUGGINS tasks, the analyses were performed with log-transformed threshold data.

\section{Results}

The left four columns of panels of Fig. 1 compare the performance of the four basic tasks (ITD, ILD, TIME, and LEVEL) between listener groups. For the lowfrequency stimulus (the upper row of panels in Fig. 1), a one-way analysis of variance indicated a statistically significant effect of listener group in the TIME task only. Subsequent pair-wise comparisons indicated higher thresholds for the ENH and EHI listeners than for the YNH listeners. For the high-frequency stimulus (the lower rows of panels), a significant effect of listener group was found in the ITD, $T I M E$, and LEVEL tasks. These results reflect higher thresholds for EHI than for ENH (ITD task); for ENH and EHI than for YNH (TIME task); and for ENH than for YNH ( $L E V E L$ task). The listener-group effect was not significant for the ILD task for either stimulus type. The figure also shows the data obtained in the supplementary experiments (FRES, FDISC, and HUGGINS tasks). Significant listenergroup effects were found in the HUGGINS task and the FDISC task (for the highfrequency stimulus only).

The thresholds of individual listeners are compared between pairs of tasks in Fig. 2. The results show statistically significant positive correlations for the ITD$I L D$ and ILD-LEVEL pairs. The partial correlation coefficients by controlling the effect of age (YNH versus ENH/EHI) were also significant. A significant partial correlation coefficient was also found for the ITD-TIME pair with the high-frequency stimulus. 


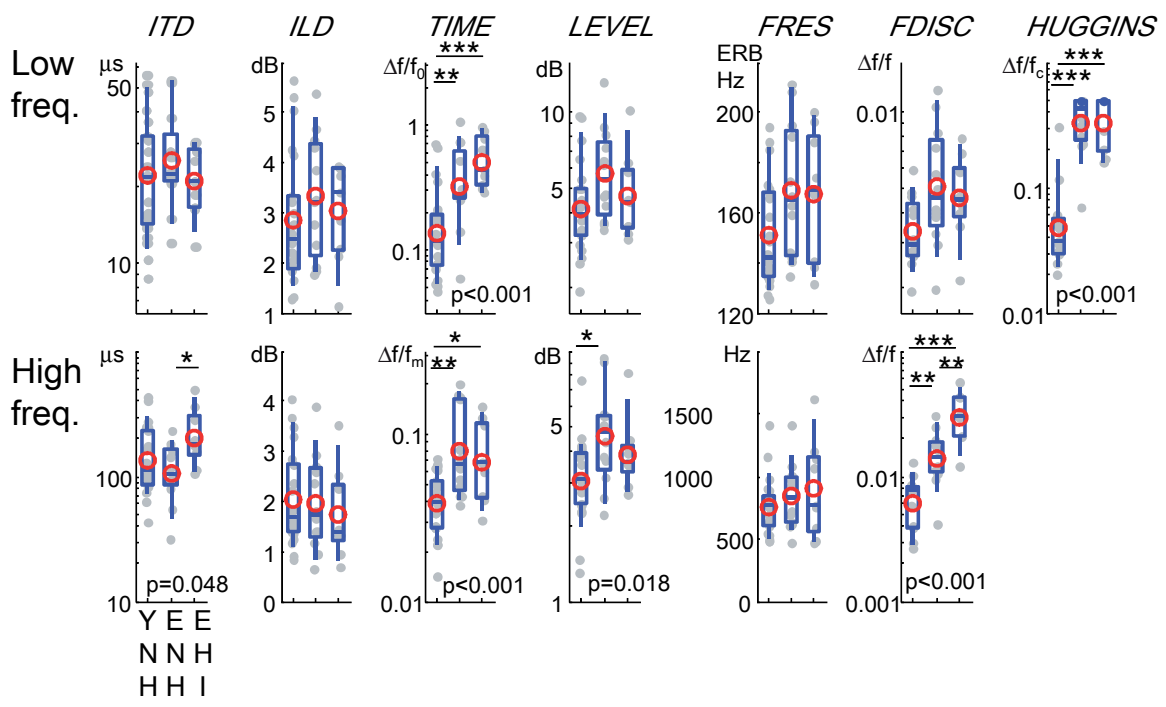

Fig. 1 Comparisons of thresholds among listener groups. Each column of panels represents one task, as labelled. The three columns of data within a panel correspond to, from left to right, YNH, $\mathrm{ENH}$, and EHI, respectively. The 10th, 25th, 50th, 75th, and 90th percentiles are shown by the box and whisker plot. The red circle shows the mean, and the grey dots represent individual listeners' data. The task for which a significant effect of listener group was revealed by one-way analysis of variance is indicated by the $p$-values. Asterisks indicate group pairs for which a significant difference was indicated by a post hoc tast with Tukey's honestly significant difference criterion $\left({ }^{*} p<0.05 ;{ }^{*} p<0.01 ; * * * p<0.001\right)$

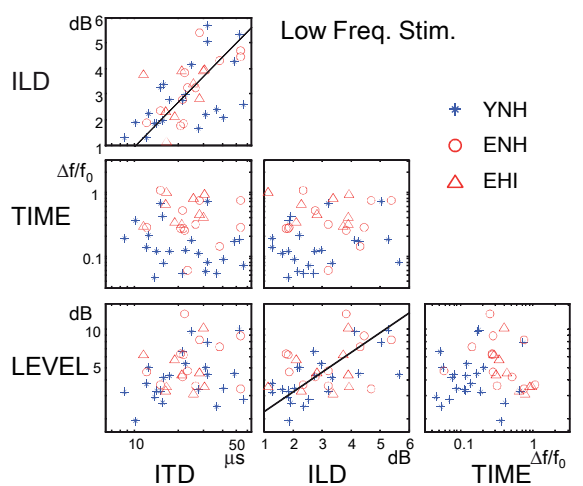

a

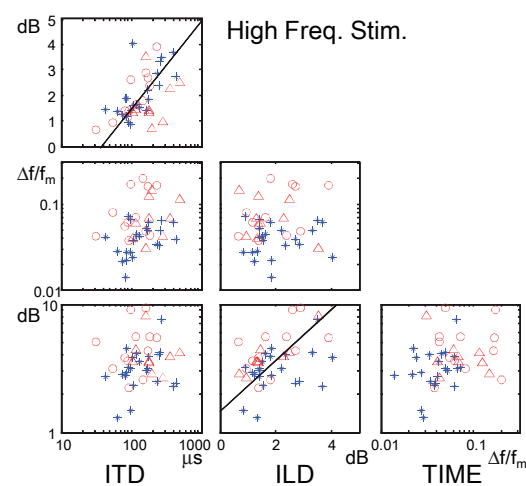

b

Fig. 2 Comparisons of individual listeners' thresholds between tasks for a low-and $\mathbf{b}$ high- frequency stimuli. Significant correlations were marked by straight lines obtained by orthogonal regressions. See also Table 2 
Table 2 Pearson's correlation coefficients and $p$-values (in parentheses) for the data shown in Fig. 2. The second line of each entry indicates the values when the effect of age was partialled out. Significant correlations $(p<0.05)$ were marked as italic characters

\begin{tabular}{|c|c|c|c|c|}
\hline & & ITD & ILD & TIME \\
\hline \multirow[t]{3}{*}{ Low Freq. } & ILD & $\begin{array}{l}0.59(<0.001) \\
0.59(<0.001)\end{array}$ & & \\
\hline & TIME & $\begin{array}{l}-0.10(0.520) \\
-0.17(0.283)\end{array}$ & $\begin{array}{l}0.16(0.318) \\
0.09(0.572)\end{array}$ & \\
\hline & LEVEL & $\begin{array}{l}0.28(0.069) \\
0.27(0.079)\end{array}$ & $\begin{array}{l}0.53(<0.001) \\
0.52(<0.001)\end{array}$ & $\begin{array}{l}0.03(0.825) \\
-0.16(0.316)\end{array}$ \\
\hline \multirow[t]{3}{*}{ High Freq. } & ILD & $\begin{array}{l}0.55(<0.001) \\
0.56(<0.001)\end{array}$ & & \\
\hline & TIME & $\begin{array}{l}0.30(0.053) \\
0.33(0.031)\end{array}$ & $\begin{array}{l}0.08(0.632) \\
0.16(0.325)\end{array}$ & \\
\hline & LEVEL & $\begin{array}{l}0.12(0.436) \\
0.12(0.462)\end{array}$ & $\begin{array}{l}0.43(0.004) \\
0.52(<0.001)\end{array}$ & $\begin{array}{l}0.28(0.064) \\
0.08(0.616)\end{array}$ \\
\hline
\end{tabular}

\section{Discussion}

Elderly listeners (ENH and EHI) showed higher thresholds than young listeners (YNH) in the TIME task (with both low- and high-frequency stimuli) and the $H U G$ GINS task. This confirms earlier findings that the sensitivity to temporal structure declines without accompanying elevated audiometric thresholds (e.g., Hopkins and Moore 2011). Thresholds for the LEVEL task also tended to be higher in the elderly than the young, consistently with previous finding (e.g., He et al. 1998). Despite these consistent declines in the performance of the monaural tasks with age, the present study failed to find significant age effect in the lateralization tasks (ITD and $I L D)$.

The thresholds for the ILD and LEVEL tasks correlated for both the low- and high-frequency stimuli. This confirms the results of our earlier study (Ochi et al. 2014), suggesting that the efficiency of level coding in the auditory periphery is a major factor accounting for inter-individual variation of ILD sensitivity. The ITD task showed a correlation with the TIME task for the high-frequency stimulus; when the factors of age and hearing-impairments were controlled. This again is consistent with the finding of our earlier study (Ochi et al. 2014), suggesting that a listener's ITD sensitivity is well accounted for by the listener's sensitivity to temporal (envelope) structure.

Despite a relatively large number of participants and greater range of threshold values in the TIME task, however, we failed to find a correlation between the ITD and TIME tasks for the low-frequency stimulus. The thresholds for the HUGGINS task showed a significant positive correlation with those for the TIME task ( $r=0.50$, $p=0.004)$, but not with those for the ITD task $(r=0.11, p=0.553)$. This suggests that the performances of the TIME and HUGGINS tasks capture inter-individual variation of the efficiency of temporal-structure processing, but that of the ITD task is determined primarily by other factors. The HUGGINS and ITD tasks are similar 
in that both require interaural comparison of temporal-structure information, but differ in the perceptual domain in which listeners are expected to respond (i.e., pitch versus laterality).

It should be noted, however, that the positive correlation found for the TIME and HUGGINS tasks was due predominantly to the consistent age effect in the both tasks (see Fig. 1). When the effects of age were partialled out, the correlation coefficient was not significantly different from zero ( $r=-0.24, p=0.191)$, implying that the positive correlation was due to underlying non-temporal factors that are sensitive to aging. A candidate for such a factor is frequency selectivity. It has been argued that (intermediately) resolved frequency components of the SSMC could contribute to the performance of the TIME task (Micheyl et al. 2010). Peripheral frequency resolution could influence the representation of interaural correlation across a frequency axis, which is the basis for the Huggins pitch. Indeed, ERB was significantly correlated with thresholds for the TIME and HUGGINS tasks (ERBTIME: $r=0.30, p=0.047$; ERB-HUGGINS: $r=0.35, p=0.050$; the effects of age were not partialled out).

Acknowledgments This study was supported by an internal basic research fund of NTT Corporation.

Open Access This chapter is distributed under the terms of the Creative Commons AttributionNoncommercial 2.5 License (http://creativecommons.org/licenses/by-nc/2.5/) which permits any noncommercial use, distribution, and reproduction in any medium, provided the original author(s) and source are credited.

The images or other third party material in this chapter are included in the work's Creative Commons license, unless indicated otherwise in the credit line; if such material is not included in the work's Creative Commons license and the respective action is not permitted by statutory regulation, users will need to obtain permission from the license holder to duplicate, adapt or reproduce the material.

\section{References}

Bernstein LR, Trahiotis C (2002) Enhancing sensitivity to interaural delays at high frequencies by using "transposed stimuli". J Acoust Soc Am 112(3):1026-1036

Cramer EM, Huggins WH (1958) Creation of pitch through binaural interaction. J Acoust Soc Am 30(5):413-417

He N, Dubno JR, Mills JH (1998) Frequency and intensity discrimination measured in a maximum-likelihood procedure from young and aged normal-hearing subjects. J Acoust Soc Am 103(1):553-565

Hopkins K, Moore BC (2011) The effects of age and cochlear hearing loss on temporal fine structure sensitivity, frequency selectivity, and speech reception in noise. J Acoust Soc Am 130(1):334-349

Micheyl C, Dai H, Oxenham AJ (2010) On the possible influence of spectral- and temporal-envelope cues in tests of sensitivity to temporal fine structure. J Acoust Soc Am 127(3):1809-1810

Moore BC, Sek A (2009) Development of a fast method for determining sensitivity to temporal fine structure. Int J Audiol 48(4):161-171 
Ochi A, Yamasoba T, Furukawa S (2014) Factors that account for inter-individual variability of lateralization performance revealed by correlations of performance among multiple psychoacoustical tasks. Front Neurosci 8. doi:10.3389/fnins.2014.00027

Patterson RD, Nimmo-Smith I, Weber DL, Milroy R (1982) The deterioration of hearing with age: frequency selectivity, the critical ratio, the audiogram, and speech threshold. J Acoust Soc Am 72(6):1788-1803 